\title{
Relapse rate of nephrotic syndrome in the time of COVID-19
}

\author{
Jérôme Harambat $^{1}$ (D) - Lise Allard ${ }^{1} \cdot$ Astrid Godron-Dubrasquet $^{1}$ \\ Received: 4 October 2020 / Accepted: 6 October 2020 / Published online: 30 October 2020 \\ (C) IPNA 2020
}

\section{Dear Editors,}

We read with interest the reports of new onset or recurrence of idiopathic nephrotic syndrome (NS) triggered by SARS-CoV-2 infection recently published in Pediatric Nephrology [1, 2].

Children are considered at lower risk of severe novel coronavirus disease 2019 (COVID-19) compared to adults but data on the impact of the disease on specific chronic conditions such as NS remain scarce. An international survey showed that children taking immunosuppressive medication for various chronic kidney diseases including patients with NS appear to have a mild clinical course of COVID-19 [3]. Moreover, it is well recognized that viral infections may trigger relapses of NS, and in those children with steroid-sensitive NS, a viral upper respiratory tract infection may result in relapses in over 50\%. Whether SARS-CoV-2 infection has the potential to precipitate NS relapses and the impact of the COVID-19 pandemic on the relapse rate of NS have yet to be determined.

As part of the worldwide pandemic of COVID-19, France imposed a national lockdown from March 17, 2020, and telemedicine was promptly implemented to manage patients with NS in our institution. The period has been hit with daily phone calls or emails from our patients with concerns as to how the situation will affect them. Most questions were related to patients' risk of infection, but many parents were also anxious about delays in diagnosis and treatment of relapses. To address this issue, we used Electronic Health Records (EHR) that include reporting of routine patient health data as well as electronic/phone communications

Jérôme Harambat

jerome.harambat@chu-bordeaux.fr

1 Pediatric Nephrology Unit, Centre de Référence Maladies Rénales Rares SoRare, Bordeaux University Hospital, Bordeaux, France between patient caregivers, physicians, and administrative support. Relevant clinical data was retrospectively extracted from EHR for 24 weeks from March 16 to August 30, 2020, and compared to a similar 24-week period in 2019 (March 18 to September 1, 2019). Out of 111 children with idiopathic NS (72 boys, median age at diagnosis 4 years, $87 \%$ experienced at least one relapse) currently followed in our center, 39 relapses were reported during the 12 -week period in $2020(0.78$ relapse/patient-year; 95\% confidence intervals (CI) 0.69 0.87). The relapse rate was lower, although not significantly, compared to that of the 2019 cohort of 105 children (0.91 relapse/patient-year; 95\% CI 0.75-1.04). No patients from the 2020 cohort except two were tested for SARS-CoV-2 because they did not develop any viral symptom at the time of relapse, the remaining two were negative. There was no evidence of a difference in relapse severity between the two periods.

Given the heavy lockdown established in the country until May 11, 2020, and the low circulation of SARS-CoV-2 in Southwest France at the peak of the epidemic's first wave, the generalizability of our findings is questionable. However, our report does not support a high risk of relapse among children with idiopathic NS managed during the COVID-19 pandemic. Conversely, our data might suggest little influence of the lockdown and following restrictions on ensuring adequate follow-up and short-term outcome of childhood NS.

\section{Compliance with ethical standards}

Conflict of interest The authors declare that they have no conflict of interest.

\section{References}

1. Enya T, Sugimoto K (2020) SARS-CoV-2 infection associated with the recurrence of nephrotic syndrome in a Japanese boy. Pediatr Nephrol. https://doi.org/10.1007/s00467-020-04782-2 
2. Alvarado A, Franceschi G, Resplandor E, Sumba J, Orta N (2020) COVID-19 associated with onset nephrotic syndrome in a pediatric patient: coincidence or related conditions? Pediatr Nephrol. https:// doi.org/10.1007/s00467-020-04724-y

3. Marlais M, Wlodkowski T, Vivarelli M, Pape L, Tönshoff B, Schaefer F, Tullus K (2020) The severity of COVID-19 in children on immunosuppressive medication. Lancet Child Adolesc Health 4: e17-e18

Publisher's note Springer Nature remains neutral with regard to jurisdictional claims in published maps and institutional affiliations. 